\title{
To burst, or rather, not to burst
}

TO THE EDITOR - The article by Swadlow and Gusev ${ }^{1}$ shows the impact of thalamic spike-bursts on neocortical targets. Recordings performed in the alert state during hippocampal theta activity, followed by high-voltage irregular activity (HVIR, a sign of drowsiness and sleep), demonstrated single-spike activity during the wake state; spike-bursts were common when animals fell into drowsiness or sleep. In the article, Fig. 1a depicts two thalamic neurons; VB1 displayed no spike-burst during waking and VB2 fired just one spike-burst throughout the entire epoch of waking. This result was expected, because intracellular studies on lateral geniculate (LG) relay neurons demonstrate that these neurons are hyperpolarized during slow-wave sleep, and depolarized during brain-active states of waking and REM sleep $^{2}$. LG and other thalamocortical neurons fire spike-bursts during slow-wave sleep and single spikes during waking because the low-threshold $\mathrm{Ca}^{2+}$ conductance that generates spike-bursts in thalamic neurons is inactivated during membrane depolarization in wakefulness, and is de-inactivated during hyperpolarization in slow-wave sleep (a notion that students acquire during their first contact with thalamic electrophysiology).

To my surprise, in the News \& Views piece ("A wake-up call from the thalamus" 3 ) that was associated with the article, S. Murray Sherman made the blunt statement that Swadlow and Gusev "offer powerful new evidence that burst mode is a normal firing mode of thalamic neurons during the waking state..."3. This is exactly contrary to data illustrated in Fig. 1 of that paper ${ }^{1}$ (see above). Waking is a behavioral state characterized by increased reactivity and highly adapted responses. Physiologically, waking is defined by activation processes in thalamocortical systems; a major feature of brain activation is the tonic depolarization of thalamic relay neurons, which prevents the occurrence of spike-bursts. Recently, researchers conducting a study on LG thalamic neurons in cats implanted with chronic recording electrodes ${ }^{4}$ also concluded that, "during wakefulness, $<1 \%$ of action potentials were associated with bursting," and found a "negative relationship between attention and bursting." Therefore, burst mode is not a normal firing mode of thalamocortical neurons during waking. Indeed, why would a "wake-up call"3 be needed if the animal were already awake? Somnolence and even drowsiness are associated with a slight hyperpolarization of thalamocortical neurons-and spike-bursts can be generated during this time- - but these states do not represent active wakefulness.

Why hark back to this issue? Simply because some readers may take for granted such lapidary statements ("burst mode is a normal firing mode of thalamic neurons during the waking state" 3 ), without qualifications of the behavioral state and the incidence of spike-bursts. It is possible that, during waking, occasional spikebursts are evoked in LG neurons by abnormally synchronous afferent volleys, such as following electrical stimuli applied to the optic nerve. However, this scenario has never been tested experimentally using intracellular recordings during opposing behavioral states of sleep and waking.

Instead of formulating claims that do not fit with data, the promoter of a 'wakeup call' hypothesis should finally try to provide sound evidence, at the intracellular level, for spike-bursts during EEG activation (when thalamocortical neurons are depolarized); such experiments are technically possible ${ }^{2}$. Then, and only then, the plethora of hypotheses from the previously postulated 'scanning attention' (under anesthesia!), to the more recent 'wake-up call,' will be vindicated.

\section{Mircea Steriade}

Laboratory of Neurophysiology, School of Medicine, Laval University, Quebec G1K 7P4, Canada

e-mail: mircea.steriade@phs.ulaval.ca

1. Swadlow, H. A. \& Gusev A. V. Nat. Neurosci. 4, 402-408 (2001).

2. Hirsch, J. C., Fourment, A. \& Marc, M. E. Brain Res. 259, 308-312 (1983).

3. Sherman, S. M. Nat. Neurosci. 4, 344-346 (2001).

4. Weyand, T. G., Boudreaux, M. \& Guido, W. J. Neurophysiol. 85, 1107-1118 (2001).
}

We welcome short letters on matters arising from previous papers in Nature Neuroscience or on other topics of widespread interest to the neuroscience community. Because space in this section of the journal is limited, priority is given to short (fewer than 500 words), well-written letters addressing the most topical issues. Typically, new data are not presented in this section, although they may occasionally be allowed at the discretion of the editors. Letters concerning material previously published in Nature Neuroscience are usually sent to the authors of the original piece for their comments and/or a formal reply. References, if absolutely necessary, should be restricted to ten or fewer. Letters may be edited for brevity and clarity. 